\title{
Evaluación de Trichoderma harzianum Rifai como inductor de resistencia a la pudrición blanca Sclerotium rolfsii. Sacc de la caraota (Phaseolus vulgaris L.) bajo condiciones controladas
}

\section{Evaluation of Trichoderma harzianum Rifai as resistance inducers against white rot Sclerotium rolfsii. Sacc in bean (Phaseolus vulgaris L.) under controlled conditions}

\author{
Jiménez María A ${ }^{1 *}$, Arcia M. Asdrubal Arcia ${ }^{2}$, Catalina Ramis ${ }^{2}$, Yreny de Faría ${ }^{2}$
}

\section{Datos del Articulo \\ ${ }^{1}$ Universidad Centroccidental Lisandro Alvarado, Laboratorio de Fitopatología, estado Lara Venezuela. \\ ${ }^{2}$ Universidad Central de Venezuela, estado Aragua, Venezuela. \\ mjimeneztamayo@gmail.com. \\ arcia.asdrubal@gmail.com \\ ramis.catalina@gmail.com \\ efariay@agr.ucv.ve \\ elf. (0251) 259.2490 - 259.2493. Fax. (0251) 259.2571 \\ *Dirección de contacto: Maria A. Jiménez. Apartado 4003001 Lara. Venezuela Telf. (0251) 259.2490 - 259.2493. Fax. (0251) 259.2571 \\ E-mail: mjimeneztamayo@gmail.com}

\section{Palabras clave: \\ Resistencia inducida, \\ incidencia final de la enfermedad, peroxidasas.}

J Selva Andina Res Soc. 2013; 4(1):31-41.

\section{Historial del artículo.}

Recibido Noviembre, 2012

Devuelto Junio 2013

Aceptado Julio, 2013

Disponible en línea, Agosto, 2013

Editado por:

Selva Andina

Research Society

\section{Resumen}

Para evaluar el efecto de Trichoderma harzianum como inductor de resistencia a la pudrición blanca producida por Sclerotium rolfsii. sobre plantas de caraota, (Phaseolus vulgaris L.) se determinó el comportamiento de las enzimas peroxidasas (POX) y la incidencia final de la enfermedad (IE), en un ensayo bajo condiciones de umbráculo. El hongo inductor, fue aplicado con una concentración de $1 \times 10^{7}$ conidias $/ \mathrm{mL}$ a semillas de P. vulgaris cv. "Magdaleno" (cvM) y cv "Tacarigua" (cvT). Tres días posteriores a la aplicación, se colocaron 3 esclerocios del patógeno, en el cuello de las plantas y se dispusieron bajo un diseño completamente al azar con cuatro tratamientos y cuatro repeticiones. Las muestras de las hojas fueron sometidas al análisis de las enzimas, desde el día 1 al día 7 post-inoculación. La separación de las isoenzimas se realizó mediante electroforesis en geles de poliacrilamida. Se encontró que la actividad POX se indujo a las 24 horas posteriores a la aplicación de T. harzianum y se mantuvo hasta el día siete. La actividad de la enzima fue inducida por el patógeno, al quinto día en el cvT y al segundo día en el cvM, revelando que potencialmente puede ser considerado un material resistente. Los resultados coincidieron con los encontrados en el ensayo bajo condiciones de umbráculo ya que la IE disminuyó a 46\% en el cvM demostrando la importancia de realizar estudios bioquímicos para determinar el momento y frecuencia de aplicación del inductor, para diseñar un manejo integrado de la enfermedad a lo largo del cultivo.

(C) 2013. Journal of the Selva Andina Research Society. Bolivia. Todos los derechos reservados.

Abstract
To evaluate the effect of Trichoderma harzianum as resistance inducers against white rot Sclerotium rolfsii. in bean
(Phaseolus vulgaris L.) peroxidase enzymes (POX) and Final Disease Incidence (DI) an experiment was set, using
the fungi in greenhouse conditions. The fungus inductor, under the commercial formulation was applied at a
concentration of $1 \times 10^{7}$ conidia / $\mathrm{mL}$ to seeds of P. vulgaris cv. "Magdaleno" (cvM) and cv "Tacarigua" (cvT). Three
days after the application was placed 3 sclerotia of the pathogen in the crown of the plants and placed in a completely
randomized design with four treatments and four replications. Samples leaf to analysis of enzymes; from day 1 to day
7 post-inoculation were subjected. The separation of the isoenzymes was performed by polyacrylamide gel
electrophoresis. The activity was induced POX 24 hours after the application of $T$. harzianum and maintained until day
seven. The enzyme activity by the pathogen was induced, fifth day in the cvT and second day at the cvM, revealing
that potentially can be considered a resistant material. The results were consistent with those found in the assay under




\section{Introducción}

La caraota (Phaseolus vulgaris L.) es un componente de gran importancia en la dieta de la población latinoamericana, por ser la leguminosa que representa la principal y más económica fuente de proteína $(22 \%)$ y calorías $(390 \mathrm{cal} / 100$ g), destinada al consumo humano (Morros 2000). El cultivo en Venezuela, tiene gran jerarquía en los programas de rotación, por su capacidad de fijar el nitrógeno atmosférico, como mejorador de la fertilidad de los suelos y abono verde (Ramírez et al 1998).

A pesar de ser un cultivo con un alto potencial productivo, una diversidad de factores afecta negativamente su productividad, lo que conllevan a un bajo rendimiento del cultivo, entre ellos destaca la pudrición blanca causada por el hongo Sclerotium rolfsii. Sacc., que, provoca inicialmente en la planta clorosis leve, seguida de una marchitez y colapso generalizado en estados avanzados, donde puede observarse el micelio algodonoso que forma esclerocios como principal forma de diseminación. Con miras a establecer un combate efectivo del hongo, enmarcado dentro del manejo integrado de la enfermedad, se han usado fungicidas (Rondón et al 1995), prácticas agronómicas como el empleo de diferentes tipos de suelos (Díaz-Polanco y Salas 1975) frecuencia de riego, abonos verdes y humus (Flores et al 1993, Ramírez et al 1998) y el control biológico, con diferentes especies de Trichoderma, evaluando su capacidad antagonista, combinada con otras prácticas tanto bajo condiciones de umbráculo como de campo (Latiegue, 1990, Arcia \& Rodríguez 1993, García \& López 1993). Esta alternativa ha logrado reducciones significativas en la densidad de esclerocios en suelo (Harman et al 2004), proporcionado un mayor crecimiento radical y foliar, mejora la absorción de nutrientes, facilitando la germinación de semillas e incrementando su desarrollo $\mathrm{y}$ producción (Kleifeld \& Chet 1992, Monte 2001). Se ha evaluado su efecto como inductor de mecanismos defensivos locales y sistémicos, ya que cepas del antagonista son capaces de establecer una interacción con las raíces (Benitez et al 2004, Harman et al 2004) conduciendo a la acumulación de moléculas de defensa, que incluyen: la producción de peroxidasas y compuestos fenólicos (Howell \& Puckhaber 2005, Howell 2003), la acumulación de hidrolasas, quitinasas, glucanasas, deposición de polímeros estructurales, como la lignina y glicoproteínas ricas en hidroxiprolina (Whipps et al 2001, Yedidia 2003). Estos mecanismos de inducción de resistencia (IR) son similares a la respuesta hipersensible (RH) resistencia sistémica adquirida (RSA) y resistencia sistémica inducida (RSI), a nivel molecular inducen la expresión de las enzimas peroxidasas consideradas como parte de una respuesta de defensa no específica iniciada posterior al ataque de un fitopatógeno, confiriéndole resistencia. 
Algunas modificaciones de pared, durante el proceso de colonización del hongo biocontrolador, represión del agente causal de enfermedades en plantas pueden desempeñar distintas funciones en los complejos procesos de reconocimiento como de posible reacción defensiva (Benitez el al 2004). Los cambios detectados en estas enzimas pueden revelar la sensibilidad que poseen los sistemas isoenzimáticos para mostrar que Trichoderma puede activar el nivel basal de defensa o mejorar la capacidad para activar rápida y eficazmente las respuestas defensivas celulares mediante un proceso conocido como "sensibilización" "preparación" o "priming” (Conrath et al 2002).

La evaluación de las posibles alteraciones de sistemas enzimáticos implicados en la respuesta defensiva de plantas de $P$. vulgaris en presencia del patógeno, así como, sobre la incidencia de la enfermedad, permitiría confirmar si existe un estímulo eficaz, por parte de este hongo y constituiría una plataforma para las investigaciones destinadas a resolver la problemática que limita la producción del cultivo.

Con base a este marco referencial, la presente investigación se planteó como objetivo evaluar el efecto de la aplicación Trichoderma harzianum como inductor de resistencia a la pudrición blanca de la caraota (Phaseolus vulgaris L) producida por Sclerotium rolfsii bajo condiciones de umbráculo analizando el incremento de la actividad peroxidasa y su implicación con la resistencia frente a la enfermedad.

\section{Materiales y métodos}

Lugar de estudio para análisis bioquímico. El ensayo se llevó a cabo en las instalaciones del campo experimental EXPERTA y del laboratorio de Genética Molecular del CIBA- UCV.

Obtención del patógeno. La cepa de Sclerotium rolfsii utilizada, provino de la Colección del laboratorio de EXPERTABIOL y los esclerocios necesarios para la ejecución del ensayo, fueron obtenidos a partir del cultivo del hongo fitopatógeno en medio de cultivo papa dextrosa agar (PDA) al $1.5 \%$, pH 6.5 en cajas Petri de 90 $\mathrm{mm}$ de diámetro esterilizadas en autoclave durante 20 minutos a 15 libras de presión $\left(121^{\circ} \mathrm{C}\right)$ e incubadas a temperatura ambiente bajo condiciones de laboratorio, (con una temperatura promedio de $\pm 28^{\circ} \mathrm{C}$ y una Humedad Relativa de $\pm 65 \%$ ) hasta la formación de los esclerocios aproximadamente 12 días.

Se sembraron semillas de caraota (P. vulgaris) cv. "Magdaleno" (cvM) y cv "Tacarigua" (cvT) sin manchas, decoloraciones o lesiones sobre su superficie y pertenecientes al Banco de Germoplasma del INIA- CENIAP Maracay.

Como inductor de resistencia se utilizó el producto comercial Tricobiol $^{\circledR}$ (Agrobica C.A) que se aplicó mediante la técnica de inmersión propuesta por Fernández-Larrea (2001), que consiste en sumergir semillas sanas durante 20 minutos en una

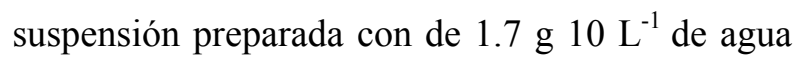
destilada esterilizada (ADE), se ajustó a una concentración final de $1 \times 10^{7}$ conidias $/ \mathrm{mL}^{-1}$. Seguidamente, se retiraron y colocaron sobre papel adsorbente esterilizado para retirar el exceso 
de agua. Las semillas testigo fueron sumergidas en una solución de ADE. Posteriormente, se sembraron en potes plásticos $(9 \times 9 \times 8 \mathrm{~cm})$, contentivos de una mezcla de sustrato y arena en proporción (1:1) previamente esterilizada con vapor, a $121^{\circ} \mathrm{C}$ durante una hora.

Una vez que emergieron los cotiledones, $T$. harzianum fue nuevamente aplicado en igual dosis que la inicial, con un volumen de $5 \mathrm{~mL}$ por planta. El hongo patógeno fue inoculado colocando 3 esclerocios por planta, en el cuello de las mismas a los tres días posteriores a la aplicación del antagonista (dpa). Las plantas testigo, sólo recibieron ADE. Una vez inoculadas, las plantas se mantuvieron bajo condiciones de umbráculo. Cada unidad experimental (UE) consistió en tres plantas por repetición y se dispusieron bajo un diseño completamente al azar, para un total de cuatro tratamientos con cuatro repeticiones por tratamiento que quedaron conformados de la siguiente forma: Testigo (Tes), Tricobiol $^{\circledR}(\mathrm{T}), S$. rolfsii $(S)$, Tricobiol ${ }^{\circledR}+$ S. rolfsii $(\mathrm{T}+\mathrm{S})$.

Preparación de las muestras para los estudios bioquímicos. Para determinar los cambios en la expresión de peroxidasas (POX) en cada muestra vegetal, se colectaron en el umbráculo 3 muestras de hojas totalmente extendidas de caraota provenientes de cada tratamiento, el 1 hasta el 7 dpa. A continuación se envolvieron en papel de aluminio, identificadas, y acondicionadas dentro de una cava con hielo seco bajo una temperatura de aproximadamente $5 \pm 2^{\circ} \mathrm{C}$ siendo transportadas hasta el laboratorio $\left(10 \pm 2^{\circ} \mathrm{C}\right)$ donde se pesaron en una balanza analítica Ohaus ${ }^{\circledR}$ Adventurer ${ }^{\mathrm{TM}}$.
Fueron resguardadas en bolsas plásticas y congeladas inmediatamente en un freezer a $-20^{\circ} \mathrm{C}$, para su almacenamiento, conservación y posterior análisis enzimático.

Extracción de las enzimas. La obtención de los extractos enzimáticos, se realizó macerando el material vegetal en nitrógeno líquido y se homogenizó con tampón de extracción conformado por $100 \mathrm{mM}$ Tris, $\mathrm{pH} 7$, al $1 \% \mathrm{y}$ Glutatión en una relación de 1:2 (tejido: tampón). Posteriormente el extracto vegetal fue centrifugado a $4^{\circ} \mathrm{C}$ durante 20 minutos a 5000 rpm. Se tomaron $15 \mu \mathrm{L}$ del sobrenadante y se colocó una gota de Azul de bromofenol para marcar el frente de la corrida. Las electroforesis se llevaron a cabo en geles de poliacrilamida bajo condiciones nativas de los diferentes extractos, de acuerdo con Laemmli (1970), sobre geles de acrilamida en un sistema de amortiguadores discontinuo, adaptado para electroforesis vertical, con dos repeticiones por peroxidasas (POX). Para las tinciones específicas, el gel se sumergió en una solución de revelado para POX, hasta la aparición de las bandas.

El estudio de sistemas de POX implicados en la respuesta defensiva de la planta por el hongo inductor sólo y combinado con el patógeno, fue establecido con base en el número, la posición e intensidad relativa de la tinción de cada banda, para ser utilizados como marcadores bioquímicos en la comparación de los tratamientos que provocaron en mayor o menor grado y la duración de la respuesta defensiva por parte del hospedante. 
Pruebas bajo condiciones de umbráculo. E1 ensayo se efectuó en bandejas en un invernadero, en las instalaciones del campo experimental EXPERTA- U.C.V.

Incidencia de la pudrición blanca de la caraota. Los tratamientos definidos para el ensayo donde se determinó la incidencia de la enfermedad (IE), fueron: plantas inoculadas con el patógeno (S) y semillas y plantas tratadas con Trichobiol $^{\circledR} \mathrm{e}$ inoculadas con el patógeno $(\mathrm{T}+\mathrm{S})$ como se describió en la prueba anterior. Para ello se prepararon 40 bandejas plásticas ( $30 \times 25 \times 14 \mathrm{~cm}$ de profundidad) con sustrato esterilizado. Se sembraron semillas de caraota tratadas con una suspensión ajustada a una concentración final de $1 \times 10^{7}$ conidias $/ \mathrm{mL}^{-1}$. Una vez que emergieron los cotiledones, se aplicó nuevamente el hongo inductor como se describió en el ensayo anterior.

El patógeno, se inoculó colocando 1 esclerocio del hongo en cada extremo de la bandeja y en el centro de la misma (equidistantes a $15 \mathrm{~cm}$ aproximadamente) y mezclándolos posteriormente con el sustrato. Todo esto se realizó 3 días posteriores a la aplicación del inductor. Cada UE consistió en una bandeja con 15 plantas c/u, separadas por una distancia de $5 \mathrm{~cm}$ con total de 5 esclerocios por UE.

Las UE's se dispusieron en un diseño en bloques al azar que incluyó 3 tratamientos para cada cultivar, con 5 repeticiones cada uno. En cada evaluación se midió la incidencia de la enfermedad (IE) considerando como número de plantas enfermas y número de plantas evaluadas para cada UE expresada en porcentaje, de acuerdo con la fórmula propuesta por Ramírez et al (1998) 35
$(\mathrm{IE})=\mathrm{N}^{\mathrm{o}}$ de plantas enfermas $/ \mathrm{N}^{\mathrm{o}}$ total de plantas $\times 100$

Análisis estadístico. Los resultados obtenidos para cada caso, se sometieron a un análisis de varianza $(\mathrm{P} \leq 0.05)$; se realizó la prueba de comparación múltiple de Tukey, para ello se utilizó el programa estadístico STATISTIX versión 8.0. con el fin de determinar los tratamientos que produjeron el menor porcentaje de plantas muertas y la menor IE por la aplicación del hongo antagonista.

\section{Resultados}

La figura 1 muestra el efecto de la aplicación de los tratamientos sobre plantas de Phaseolus vulgaris cv "Magdaleno" (cvM) y cv "Tacarigua" (cvT) respectivamente.

Los perfiles electroforéticos de extracto de hojas, tomadas del 1 al 7 día postaplicación (dpa) permitió observar los tratamientos que provocaron en mayor o menor grado y la duración de la respuesta defensiva por parte del hospedante. En la figura 1A mostró que la respuesta defensiva se observó en el cvM, a las 24 horas (1dpa) y aumento a las 48 horas (2 dpa) detectó dos isoformas de la enzima en aquellas plantas donde se aplicó el tratamiento Sclerotium rolfsii (S). Una isoforma se indujo con mayor magnitud al día 3, se mantuvo durante los días 5 y 6 e incrementó su intensidad el 7dpa, dónde se observaron nuevamente 2 isoformas. Aquellas plantas donde se aplicó Trichobiol ${ }^{\circledR}(\mathrm{T})$ la respuesta defensiva se observó al 1dpa revelando que el hongo biocontrolador puede inducir la expresión de dicha enzima, a partir de las 24 horas posteriores a su aplicación. Se detectaron dos isoformas con un 
incremento de la intensidad. En el tratamiento donde se aplicaron el hongo antagonista con el patógeno $(\mathrm{T}+\mathrm{S}$ cvM) la expresión enzimática se observó al 3 dpa y se mantuvo hasta los 7 dpa, de esta manera la respuesta permanece por mayor tiempo cuando se aplica el inductor biológico y posteriormente se expone a la planta frente al patógeno. Sin embargo, este último no logró que la actividad de la enzima se expresara a las 24 o 48 horas cuando se le comparó con los tratamientos $\mathrm{S}$ y T.

Fig1 Plantas de Phaseolus vulgaris tratadas con Trichoderma harzianum e inoculadas con S. rolfsii. Patrón izoenzimático de peroxidasas en hojas correspondientes a: cv "Magdaleno" (A) y (B) cv "Tacarigua" (Tes) plantas testigo. ( $\mathrm{S}+\mathrm{T}$ ) plantas inoculadas con S. rolfsii tratadas previamente con T. harzianum. (T) plantas tratadas con $T$. harzianum y (S) plantas inoculadas con S. rolfsii. Muestras tomadas del 1 al 7 día pos aplicación (dpa).
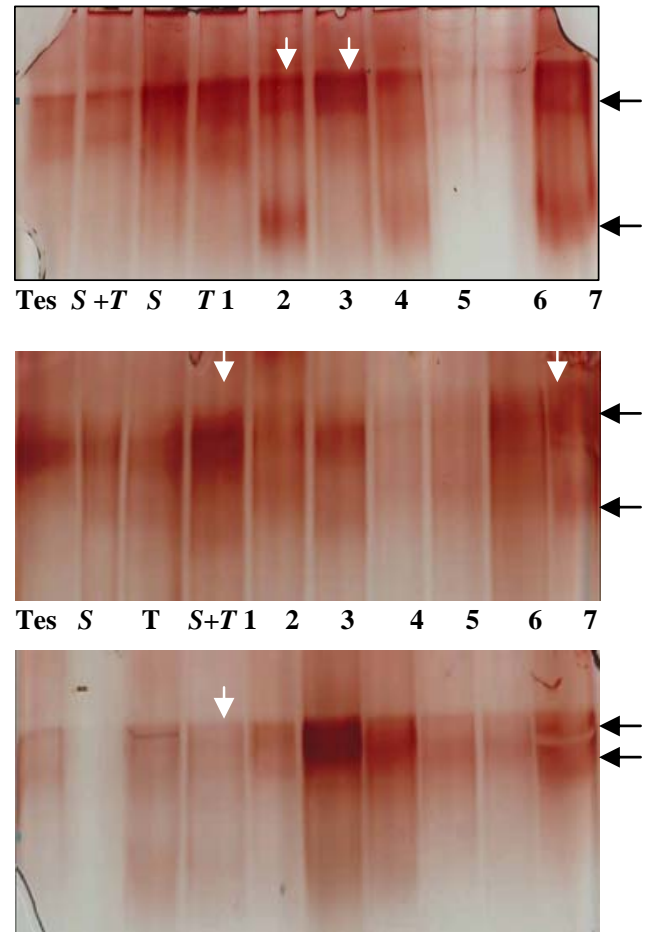

(A)

La figura 1B muestra el efecto de los tratamientos sobre la actividad de las POX en las plantas cvT donde se observó que la respuesta inducida se presentó a los 4dpa y aparecen dos isoenzimas el 5 dpa al aplicar S, denotando una respuesta tardía cuando se le comparó con el cvM. El análisis de los cambios en la expresión de las POX cuando se aplicó el tratamiento $\mathrm{T}$, evidenció la mayor expresión a los 3dpa y se mantuvo hasta el último
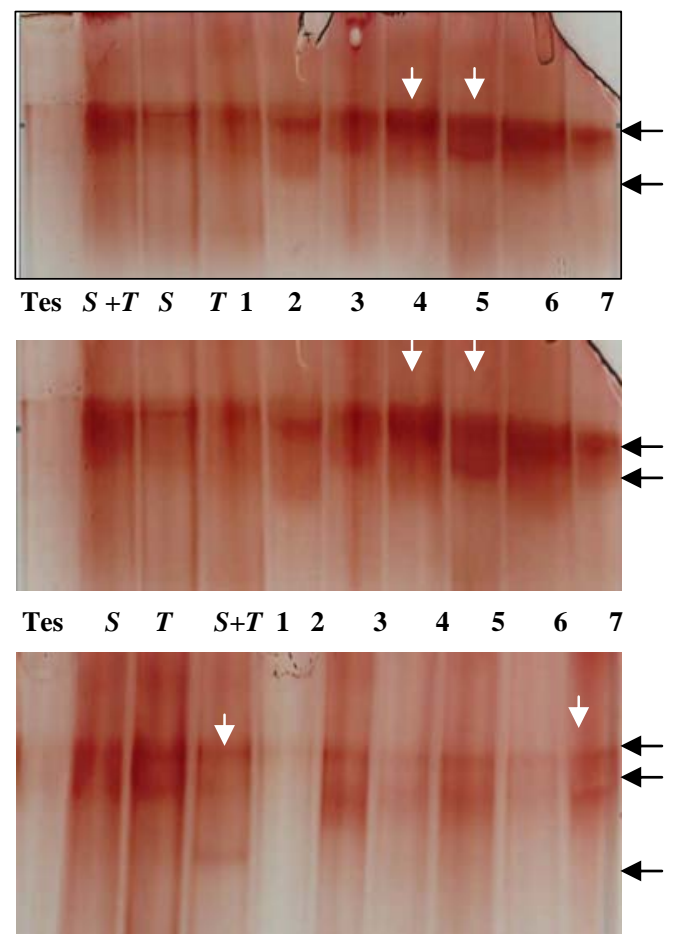

(B)

día de evaluación. $\mathrm{Al}$ aplicar ambos hongos $(\mathrm{T}+\mathrm{S})$ las planas tratadas presentaron una modificación en el comportamiento de la enzima, observándose tres bandas desde las 24 horas, de las cuales dos se mantuvieron hasta el 7 dpa, cuando se le comparó con el resto de los tratamientos.

Pruebas bajo condiciones de umbráculo. La tabla 1 muestra el efecto de los tratamientos sobre la 
incidencia (IE) de la pudrición blanca de la caraota (pb) donde se pudo observar que los mayores valores en la IE se evidenció en el tratamiento donde se aplicó el patógeno sólo en el cultivar Tacarigua ( $\mathrm{S}$ cvT) y la menor en aquellas plantas tratadas con Tricobiol $^{\circledR}$ y posteriormente inoculadas con $S$ rolfsii pertenecientes al cultivar Magdaleno ( $\mathrm{T}+\mathrm{ScvM}$ ) disminuyendo así la pb en un $46 \%$ e indicando que este genotipo logró responder frente al patógeno cuando se aplicó previamente el biocontrolador. Resultados contrarios se observaron en aquellas plantas donde se aplicó el inductor y luego el patógeno en el cultivar Tacarigua ( $\mathrm{S}+\mathrm{TcvT}$ ), mostrando que no hay inducción de la respuesta defensiva en dicho material genético, al punto en que no se evidenció diferencias significativas en la IE cuando se le comparó con las plantas inoculadas con el patógeno sólo.

Tabla 1 Efecto de los tratamientos aplicados a las plantas de caraota (Phaseolus vulgaris) sobre la incidencia de la pudrición blanca (IE) en un ensayo bajo condiciones de umbráculo.

\begin{tabular}{ll}
\hline \multicolumn{1}{c}{ Tratamento } & \multicolumn{1}{c}{ IE (\%) } \\
\hline S. rolfsii cv Tacarigua (S cvT ) & $77.280 \mathrm{a}$ \\
S. rolfsii cv Magdaleno (S cvM) & $70.380 \mathrm{ab}$ \\
T. harzianum + S. rolfsii cv Tacarigua (T+S cvT) & $63.966 \mathrm{ab}$ \\
T. harzianum + S. rolfsii cv Magdaleno (T+S cvM) & $46.520 \mathrm{~b}$ \\
\hline C.V. \% & \\
\hline
\end{tabular}

(*) Valores con la misma letra son estadísticamente iguales de acuerdo con la prueba de Tukey $(\mathrm{P}<0.05)$. El coeficiente de variabilidad indicó una variación relativamente baja para condiciones de umbráculo.

\section{Discusión}

Fue evidente que se produjo un incremento de actividad POX, cuando se aplicó el patógeno en ambos cultivares y que dicha actividad aumentó con el tiempo de inoculación. El análisis mostró además, que las primeras 48 horas representan un período crucial para la activación de los mecanismos de defensa. No obstante, al comparar la respuesta por parte de los materiales vegetales bajo estudio, fue evidente que el cvM respondió con mayor intensidad pero, la duración de la respuesta de defensa se mantuvo hasta el día siete, evidenciando que debe realizarse una nueva aplicación en este período, si se desea mantener a la planta protegida por mayor tiempo.

Las plantas de ambos cultivares tratadas con Trichobiol ${ }^{\circledR}$ mostraron un aumento en la intensidad de la banda, indicando el efecto positivo del inductor sobre la inducción de respuesta. La habilidad de cepas de Trichoderma para proteger plantas contra patógenos ha sido atribuida al efecto que provoca el antagonista sobre el agente patógeno (Harman et al 2004, Benitez et al 2004), no obstante, los perfiles electroforéticos mostraron que puede establecerse una asociación entre la raíz $\mathrm{y}$ el hongo benéfico. A nivel molecular, la respuesta resultó que en un incremento en las POX similar a la reacción hipersensible que ha sido señalada como un componente de la respuesta temprana al ataque por parte de patógenos $\mathrm{y}$ juegan un papel importante en la síntesis de lignina, que limita el avance del patógeno (Viterbo et al 2006, Woo et al 2006).

La producción de un ambiente fungitóxico, juega un papel importante en la tolerancia de la planta a 
la colonización e intento del hongo inductor por penetrar las células de las raíces restringiendo el crecimiento de la hifa dentro de los espacios intercelulares (Yedidia et al 2000), esto explicaría, la expresión de la enzima desde las 24 horas después de la aplicación del hongo benéfico.

Fue evidente que el hongo benéfico, puede afectar la resistencia de planta frente al patógeno en los cultivares evaluados, bien por la inducción de reacciones básicas de defensa inmediatamente después de aplicado o mejorando la capacidad de respuesta rápida y perduró por mayor tiempo lo que podría ayudar a disminuir la actividad del patógeno.

La actividad de la enzima fue más elevada en aquellos tejidos protegidos y que posteriormente eran inoculados con el patógeno, evidenciando que se hace necesario un lapso entre la aplicación del antagonista y el patógeno, probablemente porque se requiere de un tiempo para activar los mecanismos de defensa para la represión del patógeno, esto demuestra el carácter preventivo y no curativo de su aplicación.

Las enzimas relacionadas con la oxidación y los compuestos fenólicos, son consideradas como uno de los parámetros importantes bioquímicos para la resistencia a enfermedades y es por lo general, más alta en genotipos resistentes comparados con los susceptibles (Chakraborty \& Chatterjee 2007). Esto podría explicar por qué que el cvT estuvo en desventaja frente al patógeno cuando se le comparó con cvM.

El conocimiento generado por los perfiles electroforéticos indicó que la respuesta decae a 38 los 7 días en ambos materiales genéticos, estos resultados indican que, se pueden tratar las semillas de las plantas con el antagonista al momento de la siembra y posteriormente aplicarlo con mayor frecuencia de modo que el hongo logre asociarse y establecerse en las raíces de las plantas, y así, provoque un estímulo defensivo y adicionalmente actúe de forma directa sobre el patógeno.

Al comparar los resultados obtenidos en el ensayo bajo condiciones controladas con los mostrados en los perfiles electroforéticos, se observó que el cvM respondió rápidamente al patógeno, sin embargo, dicha respuesta no fue suficiente como para suprimir totalmente la enfermedad. No obstante, se comprobó que se puede lograr una reducción significativa de la pb hasta en un $46 \%$ en el cvM al manejar al hospedante, aportándole condiciones que le permitieron expresar su potencial metabólico lo que impidió el desarrollo del patógeno.

Los resultados evidenciaron que se hace necesario tomar en consideración no sólo la dosis, sino la frecuencia de aplicación del hongo antagonista. Fue evidente que Trichoderma puede afectar la resistencia de planta al patógeno, induciendo el nivel básico de reacciones de defensa inmediatamente después de su aplicación en el cvM, lo cual no fue observado en el cvT, probablemente porque no se estableció una relación exitosa entre el hongo inductor y las raíces de las plantas, demostrando que cada genotipo del hospedante se manifiesta de forma diferente ante las cepas del hongo ya que no hay especificidad en la respuesta tal como lo ha señalado Harman et al (2004). 
De acuerdo con el objetivo planteado y los resultados obtenidos en la investigación realizada se llegó a las siguientes conclusiones.

En los tratamientos donde se aplica Trichoderma solo, patógeno solo y ambos interactuando, se produce un aumento de la actividad peroxidasa con el tiempo de inoculación pero posteriormente decae.

El empleo de Trichoderma en las plantas de caraota como activador de los mecanismos de defensa en la planta puede ayudar a mejorar la capacidad de ésta para defenderse contra una infección subsecuente, probablemente gracias a la activación más rápida e incrementada de los genes relacionados con la defensa.

Se hace necesario conocer mediante los marcadores bioquímicos el momento de aplicación y la duración de la respuesta para diseñar un manejo integrado de la enfermedad a lo largo del cultivo, que incluya: dosis, frecuencia de aplicación, para permitir que las plantas se mantengan protegidas durante mayor tiempo, la densidad de siembra y condiciones ambientales predisponentes para la aparición de la enfermedad y generar condiciones que favorezcan la reducción de la incidencia de la enfermedad, bajo condiciones de campo.

\section{Conflictos de intereses}

Esta investigación recibió financiamiento del CDCHT /UCLA Lara y del laboratorio de Genética Molecular del CIBA- UCV, Venezuela, y no presenta conflictos de interés.

\section{Agradecimientos}

Los autores agradecen al Consejo De Desarrollo Científico Humanístico y Tecnológico (CDCHT) de la Universidad Centroccidental "Lisandro Alvarado" por financiamiento de la presente investigación bajo el código 020 AG-2008.

\section{Literatura citada}

Arcia A, Rodríguez I. Influencia de diferentes concentraciones de conidios de Trichoderma spp en el control de Sclerotium rolfsii Sacc. En: Compendio de resúmenes del XIII Congreso Venezolano de Fitopatología. 07-11 Noviembre, San Cristobal, Táchira. Venezuela. 1993; 81.

Benitez T, Rincon A.M, Limon M. C. Biocontrol mechanisms of Trichoderma strains. Int. Microbiol. 2004; 7(4):249-260.

Chakraborty MR, Chatterjee NC. Interaction of Trichoderma harzianum with Fusarium solani during its Pathogenesis and the Associated Resistance of the Host Asian J Exp Sci. 2007; 21(2):351-355.

Conrath U, Corné M, Pieterse J, Mauch-Mani B. "Priming in plant -pathogen interactions" Annu. Rev. Plant Physiol. 2002. 14: 249-270.

Fernández-Larrea O. Microorganismos antagonistas para el control fitosanitario Manejo Integrado de Plagas. Costa Rica. 2001; 62:96-100.

Flores Y, Rondón A, Soto E, Mujica Y. Control químico en condiciones in vitro de Sclerotium rolfsii Sacc causante de la pudrición blanca. En: Compendio de resúmenes del XIII Congreso Venezolano de Fitopatología. 07-11, 
Noviembre, San Cristobal, Táchira. Venezuela. 1993; 114.

Garcia E, López L. Evaluación in vitro de hongos del suelo como agentes de control biológico de Sclerotium rolfsii Sacc, causante de la pudrición basal del tomate. Resúmen $\mathrm{N}^{0} 53 . \mathrm{V}$ Jornadas Técnicas Cientificas de la Facultad de Agronomía. Rev.Fac.Agron.(LUZ) 1993; 10(1):77.

Harman G. E, Howell C. R, Viterbo A, Chet I, Lorito M. Trichoderma spp.-opportunistic avirulent plant symbionts. Nature Microbiol Rev. 2004; 2:43-56.

Howell. C. R. Mechanisms Employed by Trichoderma Species in the Biological Control of Plant Diseases: The History and Evolution of Current Concepts Southern Plains Agricultural Research Center. Plant Disease. 2003; 87(1):1-10.

Howell C, Puckhaber L. A study of the characteristics of "P" and "Q" strains of Trichoderma virens to account for differences in biological control efficacy against cotton seedling diseases. Biological Control. 2005; 33(2): 217-222.

Kleifeld O, Chet I. Trichoderma harzianum: Interaction with plants and effect on growth response. Plant and Soil. 1992; 144:267-272.

Laemmli U. 1970. Cleavage of structural proteins during the assembly of the head of bacteriophage T4. Nature. 1970; 227:680-685.

Latiegue A. Trichoderma harzianum Rifaii., Como Antagonista de Sclerotium rolfsii sacc., Agente Causal de Pudrición Basal de la Caraota Phaseolus vulgaris L.) Tesis de Maestria. UCLA. 1990; 180.

Monte E. Understanding Trichoderma: between agricultural biotechnology and microbial ecology. Int. Microbiol. 2001; 4,1-4.

Morros M. E. Cultivo de la caraota con énfasis en el estado Lara. Maracay, Ven., Instituto Nacional de Investigaciones Agrícolas. Centro de Investigaciones Agropecuarias del estado Lara. 2000; Serie D. (8): 74.

Ramírez R, Santos F, Bracho L, Sandoval Y, Castro De Rincón C. Control de Sclerotium rolfsii Sacc con fungicidas y humus. Control of Sclerotium rolfsii Sacc with fungicides and humus. Rev. Fac. Agron. (LUZ).1998; 15:534544.

Rondón A., Flores Y, Soto E, Mujica Y. Control químico in vitro y en umbráculo del hongo causante de la pudrición blanca. Rev.Fac. Agron. (LUZ).1995; 12(1):1-13.

Diaz-Polanco C, Salas de Díaz G, Saavedra F. Influencia de hongos fitopatogénicos en la reducción de la población en siembras de caraota. CIARCO V. 1975; 27-30.

Statistix for Windows version 8.0. User's Manual. Analytical Software. Tallahassee, FL, USA. Statistix. 2003.

Viterbo A, Shoresh M, Brotman E, Chet I. Towards understanding the molecular basis for induced resistance in the Trichoderma-plant interaction. In: Proceedings of the Ninth International workshop on Trichoderma and Gliocladium. Vienna, Austria, Abril. 2006; 6-8.

Whipps J. M. Microbial interactions and biocontrol in the rhizosphere. J. Exp. Bot. 2001; 52(1):487-511.

Woo S, Scala F, Ruocco M, Lorito M. The molecular biology of the interactions between Trichoderma spp., phytopathogenic fungi, and plants. Phytopathology. 2006; 96:181-185.

Yedidia I, Benhamou N, Kapulnik Y, Chet I. Induction and accumulation of pathogenesis 
related protein activity during the early stages of root colonization by Trichoderma harzianum. Plant Physiol. Biochem. 2000; 38:863-873.
Yedidia I, Shoresh M, Kerem Z, Benhamou N, Kapulnik Y, Chet I. Concomitant induction of systemic resistance to Pseudomonas syringae pv. lachrymans in cucumber by Trichoderma asperellum (T-203) and accumulation of phytoalexins. Appl. Environ. Microbiol. 2003; 69:7343-7353. 